\title{
Use of an X-linked Human Neutrophil Marker to Estimate Timing of Lyonization and Size of the Dividing Stem Cell Pool
}

\author{
E. Stephen Buescher, David W. Alling, and John I. Gallin \\ Bacterial Diseases Section, Laboratory of Clinical Investigation, National Institute of Allergy and Infectious Diseases, \\ National Institutes of Health, Bethesda, Maryland 20205
}

\begin{abstract}
In families with $\mathrm{X}$-linked chronic granulomatous disease (CGD), heterozygous females have two stable populations of polymorphonuclear leukocytes (PMN) in their blood; one normal, the other, deficient in oxygen metabolism. The two types of PMN can be distinguished by the ability or lack of ability to reduce nitroblue tetrazolium dye. The variation in the percent normal PMN among 11 CGD heterozygotes was shown to follow a binomial distribution based on eight independent trials and a chance of success of $50 \%$. This is consistent with the occurrence of $\mathrm{X}$ chromosome inactivation (lyonization) when eight embryonic founder cells for the hematopoietic system are present. Serial determinations of the percent normal PMN in individual heterozygotes showed very limited variability (standard deviations ranged from $2.0 \%$ to $5.2 \%$ ) most of which could be ascribed to experimental error. An estimate of the remaining variation (residual variance) was introduced into a well-known formula to calculate the appropriate number of pluripotent stem cells necessary to support hematopoiesis and a figure exceeding $\mathbf{4 0 0}$ was obtained. Thus, the data indicate that in humans there is a highly polyclonal system of hematopoiesis.
\end{abstract}

\section{Introduction}

Chronic granulomatous disease (CGD) ${ }^{1}$ of childhood is an inherited group of diseases recognized only in humans, in which phagocytic leukocytes have a deranged oxygen metabolism, with a consequent defect in killing of catalase-positive microorganisms (1). One form of CGD is an X-linked recessive disorder in which males are severely affected and half of their female relatives can be expected to be heterozygous carriers for the disease (2). Such carriers can almost always be shown to have two distinct populations of phagocytic cells in their blood, one with competent oxygen metabolism, the other, without. The proportions of cells belonging to the two populations are thought to be established by random $X$-chromosome inactivation early in ontogeny (2). Assays that detect competent oxygen metabolism in individual cells, such as nitroblue tetrazolium (NBT) reduction, can readily be applied to purified preparations of phagocytic cells from CGD heterozygotes to study population dynamics of polymorphonuclear leukocytes (PMN).

Address reprint requests to Dr. Gallin, National Institute of Allergy and Infectious Diseases, Building 10, Room 11N112, National Institutes of Health, Bethesda, MD 20205.

Received for publication 10 April 1985.

1. Abbreviations used in this paper: CGD, chronic granulomatous disease; NBT, nitroblue tetrazolium.

The Journal of Clinical Investigation, Inc

Volume 76, October 1985, 1581-1584

\section{Methods}

CGD heterozygotes. 11 females heterozygous for CGD were identified using the NBT reduction assay described below. All subjects were relatives of patients previously documented in the literature (1) and included seven mothers of male patients with X-linked CGD and one maternal grandmother of two brothers with X-linked CGD.

$N B T$ reduction assay. The slide NBT test of Repine et al. (3) was modified as follows: $0.5-1.0 \mathrm{ml}$ of fresh whole blood was placed on a glass slide and incubated at $37^{\circ} \mathrm{C}$ in a humidified chamber until clotting occurred $(1.5-3 \mathrm{~h})$. After the clot was gently removed the slide was rinsed in phosphate-buffered saline (PBS) to free it of erythrocytes, then covered with a solution containing $0.1 \% \mathrm{NBT}$ and $20 \mathrm{ng} / \mathrm{ml}$ phorbol myristate acetate (both obtained from Sigma Chemical Co., St. Louis, MO) in Hanks' balanced salt solution with calcium and magnesium. The slide was incubated for $15 \mathrm{~min}$ at $37^{\circ} \mathrm{C}$, then rinsed in PBS, air-dried, and counterstained with $0.25 \%$ safranine. $300-500$ cells were identified as PMN by nuclear morphology and scored as either NBT positive or NBT negative. Neutrophils from 10 normal subjects were studied using this method and showed an average of $99.6 \pm 0.18 \%$ cells that reduced NBT (range $98.5-100 \%$ ).

\section{Statistical methods.}

(a) The mean percent PMN positive for NBT in a heterozygote was assumed to follow a binomial distribution based on eight independent trials and a chance of success of $50 \%$; that is,

$$
P(100 t / 8)=(1 / 2)^{8} \sum_{x=0}^{t} 8 ! /(x !(8-x) !), \quad t=0,1, \ldots, 8,
$$

where $P(100 t / 8)$ is the probability that the mean percent $P M N$ positive for NBT will not exceed $100 t / 8$. The chance of success equal to $50 \%$ is consistent with the Lyon hypothesis (4); the number of independent trials $(n=8)$ was chosen so as to minimize the maximum vertical distance between the respective graphs of $P(100 t / 8)$ and of the corresponding observed cumulative distribution (Kolmogorov $\mathrm{D}^{+}$statistic, see Reference 5).

(b) Analysis of variance was applied to the data from 20 NBT assays (each read four times) and to five weekly assays on a pair of heterozygotes. Based on these calculations components of variance were estimated with the following results: standard deviation owing to reading error $2.31 \%$; standard deviation owing to preparative error $2.26 \%$; standard deviation owing to residual error $2.26 \%$. The latter source of variability was taken to be that owing to selection of heterogeneous clones, consistent with the clonal succession theory (6) and termed intrinsic error.

(c) The method of computer simulation described by Burton et al. (7) for erythropoiesis was simplified because it was not necessary to allow for the prolonged survival of the erythrocytes in the peripheral blood and hence for the attendant carryover effects. In fact, clones of neutrophils arising from the maturation of successive stem cells were assumed to have a carryover effect $<2 \mathrm{~d}$ in length. Accordingly, at time points separated by $2 \mathrm{~d}$, or longer, the following steady-state model was assumed: the percent PMN positive for NBT $(100 x / m)$ follows a binomial distribution

$$
f(100 x / m)=\frac{m !}{x !(m-x) !} p^{x}(1-p)^{m-x}, \quad x=0,1, \ldots, m,
$$


where $m$ is the number of clones of marrow cells simultaneously contributing to the pool of circulating neutrophils and $p$ is the chance of selecting an NBT-positive clone.

\section{Results}

11 healthy females were studied over periods of time up to 2.5 yr. Each of these subjects had both NBT-positive and NBTnegative PMN in her peripheral blood and each was a close relative of a patient with CGD. In view of the latter findings, we assumed that the subjects were CGD heterozygotes. The distribution of the mean percentage of PMN positive for NBT among the subjects is shown in the lower panel of Fig. 1. The average of the mean percentages is $53.8 \%$, which is not significantly different from $50 \%$, the predicted average if the chance were $50 \%$ that the normal X-chromosome is inactivated (Lyon hypothesis [4]).

If lyonization occurs as an independent event in each of $n$ founder cells and if it is assumed that such cells precede the pluripotent stem cell in ontogeny then the mean percent PMN positive for NBT in a heterozygote would be expected to be a binomial proportion with parameters $p=1 / 2$ and $n$. The upper panels of Fig. 1 show the mean percentages of NBT-positive PMN expressed as a cumulative distribution (solid lines) and the cumulative binomial distributions corresponding to $p=1 / 2$ and $n=4,8,16$. The binomial distribution for $n=4$ has steps that are too broad to fit the observed distribution well, whereas the binomial distribution for $n=16$ lies systematically above the observed in the range where NBT-positive PMN exceed $50 \%$. The binomial distribution for $n=8$ has neither drawback; moreover, the maximum vertical distance between this pair of distributions is the smallest of the three cases (see legend to Fig. 1).

The data collected on the 11 heterozygotes were also used to estimate the number of pluripotent stem cells actively supporting hematopoiesis. This estimate is based partly on the variation in the percent PMN positive for NBT within subjects which was found to be quite limited (standard deviations ranged from
2.0 to 5.2) compared to the variability of the mean percent PMN positive for NBT among subjects $(\mathrm{SD}=18.6)$. Examples of such data collected over extended periods of time are shown in Fig. 2. The individual series were free of linear trends when examined by regression analysis. Because of the irregular spacing of data points in time, other forms of systematic variation and serial dependence could not be ruled out with complete assurance. Nevertheless, in view of the short half-life of circulating PMN

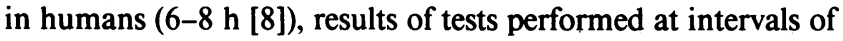
$2 \mathrm{~d}$ or longer were assumed to be independently distributed.

The number of pluripotent stem cells $(m)$ was estimated by the method described by Burton et al. (7) from the relation $m$ $=10^{4} p(1-p) / s^{2}$, where $p$ is the proportion of NBT-positive stem cells and $s^{2}$, the variance due to intrinsic variability (the latter is expressed in percent; see "Statistical methods," paragraph $b$ ). The proportion, $p$, was calculated to be 0.611 from data collected on an extensively studied heterozygote ( $\square$ in Fig. 2) and the variance, $\mathrm{s}^{2}$, to be 5.13 from pooled data collected on the same heterozygote as well as three others. Thus, $m$, the number of pluripotent stem cells was estimated to be 463 . Additional estimates of $m$ were obtained from calculated values of $p$ from the three remaining extensively studied heterozygotes and found to be 408,407 , and 467 , respectively. Conservative estimates of $m$ were also computed by assuming that all of the observed variability was due to clonal heterogeneity, i.e., that $s^{2}=21.92$ and values $108,95,95$, and 109 , respectively, were obtained.

The above results were derived by determining the number of pluripotent stem cells needed to account for the variability observed among proportions of NBT-positive neutrophils in an individual heterozygote. The inverse relationship was also studied by determining the amount of variability in such proportions of NBT-positive neutrophils that would result from the sustained production of a given number of stem cell clones. For this, a steady-state model of bone marrow PMN production was simulated by computer (see "Statistical methods," paragraph $c$ ). To afford comparison of the output from the simulation with data obtained from patients the chance of selecting a NBT-positive

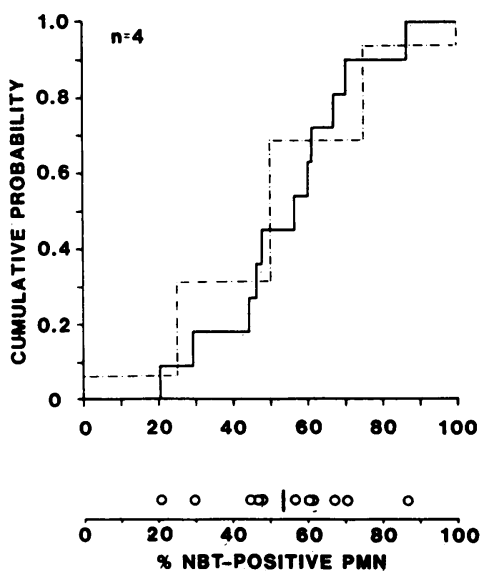

Figure 1. (Upper three panels) Cumulative distribution of mean percent PMN positive for NBT observed among 11 healthy subjects heterozygous for CGD (solid lines) is compared with corresponding cumulative distribution predicted by the binomial model, $P(100 t / n)$

$=(1 / 2)^{n} \sum_{x=0}^{t} n ! /(x !(n-x) !), t=0,1, \cdots n$, where $P(100 t / n)$ is the

chance that the mean percent PMN positive for NBT will not exceed
$100 t / n$ (dashed lines), for $n=4,8,16$. The maximum vertical distance between the respective pairs of distributions is $0.23(n=4), 0.18(n=$ $8), 0.32(n=16)$. The range of abscissas at which the maximum vertical distance occurs is 50.0-56.6 $(n=4), 50.0-56.6(n=8), 56.25-56.6$ $(n=16)$. (Lower panel). Distribution of mean percent PMN positive for NBT observed among the 11 CGD heterozygotes. 


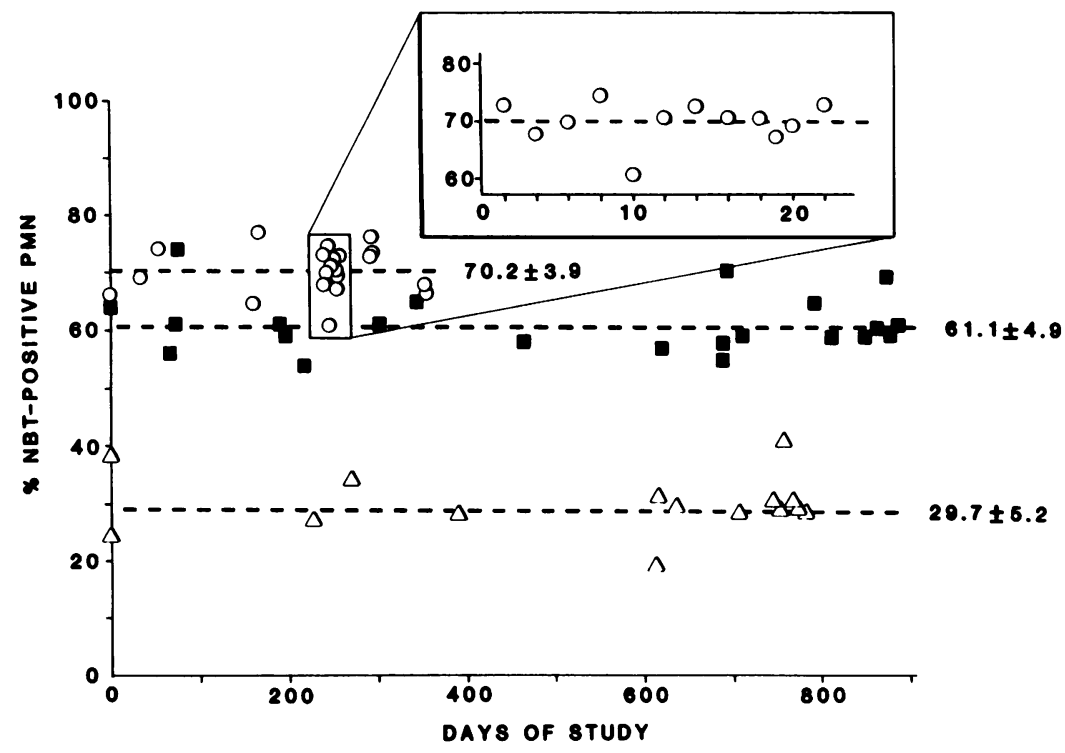

Figure 2. Percent PMN positive for NBT in serial blood samples according to day of study in each of three healthy subjects heterozygous for CGD $(0, \square$, $\Delta$ ). Dashed lines (- - ) represent best horizontal fits to the respective sets of data. The values appearing at the right end of each line denote mean \pm standard deviation. clone ( $p$ ) was taken to be 0.611 , the average for the most extensively studied heterozygote ( $\square$ in Fig. 2). Three values of the number of stem cell clones $(m)$ were chosen: 27,108 , and 432 ; these are centered (by ratio) around a conservative estimate of
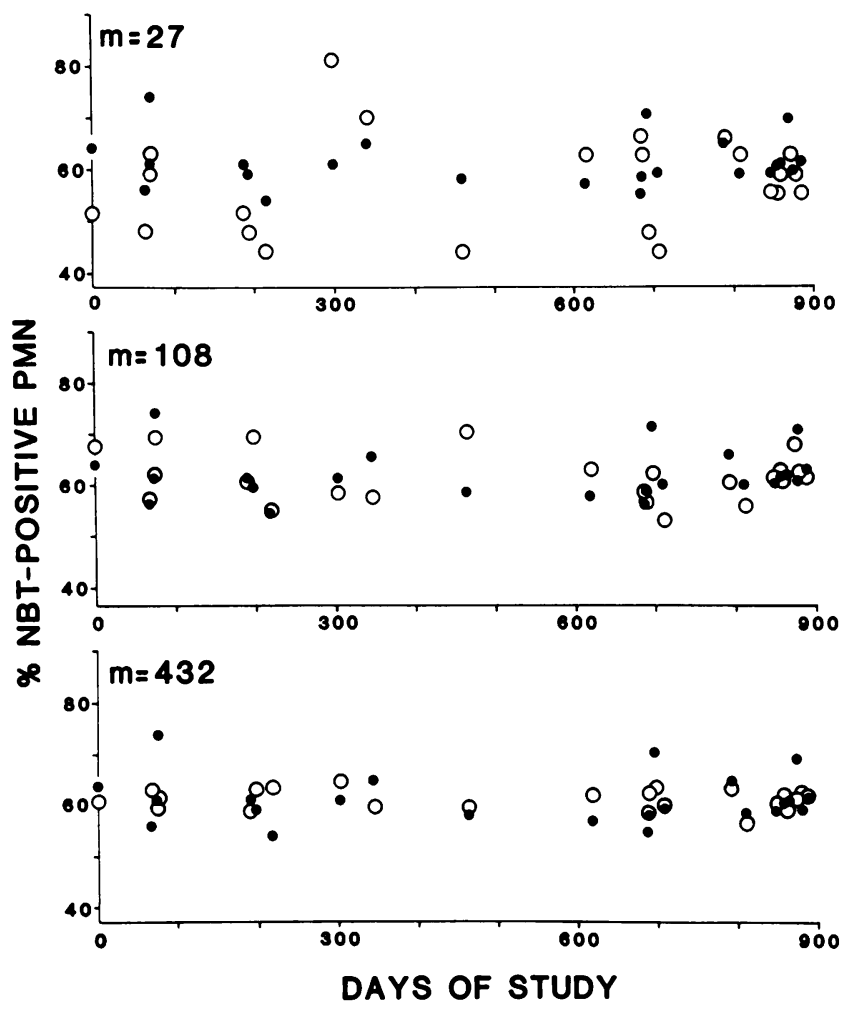

Figure 3. Computer simulation of percent PMN positive for NBT in serial blood samples collected from a CGD heterozygote.

$(\bullet)$ Observed values; (O) predicted by model.

$f(100 x / m)=\frac{m !}{x !(m-x) !} p^{x}(1-p)^{m-x}$

where $p=0.611, m=27,108,432$, and $100 x / m$ is the percent PMN positive for NBT. (See Methods.) $m$ based on a value of $s^{2}$ that included all sources of variability. The results shown in Fig. 3 indicate that, for $m=108$, there is good agreement between observed and predicted variability (standard deviations were 4.91 and 4.88 , respectively), whereas there is excess model variability for $m=27$ (standard deviation $=9.36$ ) and insufficient model variability for $m=432$ (standard deviation $=1.95$ ).

\section{Discussion}

One of the main findings of this study is the contrast between the wide variability of the percent normal PMN among CGD heterozygotes and the very limited variation of this percentage among serial determinations in individual heterozygotes. This result was obtained with the use of an accurate, reproducible assay for oxygen metabolism in individual PMN. With this assay essentially all normal PMN are positive (i.e., reduce NBT) after being stimulated with phorbol myristate acetate (9). The observed variation in the mean percent normal PMN among the 11 heterozygotes studied is consistent with the distribution expected if inactivation of one of the X-chromosomes occurred at random when about eight founder cells for the hemotopoietic system are present. This estimate is in the same range (5-10 cell stage) as those found in two studies examining human $\mathrm{X}$-linked glucose6-phosphate dehydrogenase deficiency $(10,11)$ but is lower than the figure (16 cell stage) noted in a third study (12).

In contrast, only limited variation in the percent normal PMN was observed within extensive series of determinations made on blood from individual heterozygotes. This finding, in conjunction with the clonal succession theory (6) suggests that upwards of 100 stem cell clones are simultaneously contributing to the circulating neutrophil pool. Because there is no evidence to suggest that hematopoiesis is abnormal in such heterozygotes, the inference can presumably be extended to normal subjects. Our findings do not agree with those of Burton et al. (7), who estimated that, in mice, a mean of only 10 (range 2-30) simultaneously productive clones are needed to account for the observed variation of the proportion of erythrocytes with an Xlinked trait. These authors did not, however, take account of 
sources of variability other than that originating in the clonal selection process and hence may have seriously underestimated the required number of clones. On the other hand, the discrepancy between the two sets of results may be explained in part by the difference in size of the two hematopoietic systems studied.

The data reported in this paper consist of collected series of proportions of normal PMN observed in a group of CGD heterozygotes. These data are used to draw inferences about two well-known models of cellular development, the Lyon hypothesis (4) and the clonal selection theory of hematopoiesis (6). It is generally assumed that the neutrophil first makes its appearance many generations after the occurrence of the Lyon phenomenon and at least several generations after selection has occurred from the pool of nondividing pluripotent stem cells. The proportion of normal neutrophils thus serves as an indicator of events that have occurred in the recent and remote past. Accordingly, the inferences based on such data as we have collected are conditioned by a number of unavoidable assumptions, in particular, that data collected with neutrophils can be extended broadly to hematopoiesis; hence our inferences should be treated with some caution. Nevertheless, until more direct methods of studying the Lyon hypothesis and the clonal selection theory of hematopoiesis are devised, such evidence is of interest, particularly because it relates to both models and hence draws attention to possible connections between these widely separated phenomena in cellular development.

\section{Acknowledgments}

The authors thank Mrs. Ruth Wheat, Ms. Julie Wheat, and Mrs. Heather Karp for their generous assistance to this study.

\section{References}

1. Gallin, J. I., E. S. Buescher, B. E. Seligmann, J. Nath, T. Gaither, and P. Katz. 1983. Recent advances in chronic granulomatous disease. Ann. Intern. Med. 99:657-674.

2. Mills, E. L., K. S. Rholl, and P. G. Quie. 1980. X-linked inheritance in females with chronic granulomatous disease. J. Clin. Invest. 66:332340.

3. Repine, J. E., B. Rasmussen, and J. G. White. 1979. An improved nitroblue tetrazolium test using phorbol myristate acetate-coated coverslips. Am. J. Clin. Pathol. 71:582-585.

4. Lyon, M. 1968. Chromosomal and subchromosomal inactivation. Annu. Rev. Genet. 2:31-52.

5. Lehmann, E. L. 1959. Testing Statistical Hypotheses. John Wiley \& Sons, Inc., New York. 246-247.

6. Kay, H. E. M. 1965. How many cell generations? Lancet, 2:418419.

7. Burton, D. I., J. D. Ansell, R. A. Gray, and H. S. Michlem. 1982. A stem cell for stem cells in murine hematoporesis. Nature (Lond.). 298: $562-563$.

8. Cartwright, G. E., J. W. Athens, and M. M. Wintrose. 1964. Kinetics of granulopoiesis in normal man. Blood. 24:780-803.

9. Buescher, E. S., P. V. Holland, and J. I. Gallin. 1983. Radiation induced defective oxygen metabolism leukocytes prepared for transfusion as assessed by nitroblue tetrazolium reduction. Clin. Res. 31:309A. (Abstr.)

10. Gandini, E., S. M. Garther, G. Antioni, N. Argiolas, and G. Dell'Acqua. 1968. Developmental implication of multiple tissue studies in glucose-6-phosphate dehydrogenase-deficient heterozygotes. Proc. Natl. Acad. Sci. USA. 61:945-948.

11. Nesbitt, M. N., and S. M. Gartler. 1971. The applications of genetic mosaicism to developmental problems. Annu. Rev. Genet. 5: 143-162.

12. Fialkow, P. J. 1973. Primordial cell pool size and lineage relationships of five human cell types. Ann. Hum. Genet. 37:39-48. 